\title{
Que peut-on attendre de la méthode de précipitation en milieu gélifié pour le diagnostic de la rage dans la région de Dakar
}

\author{
por G. THIERY
}

\begin{abstract}
Chargé depuis 1954 du diagnostic de la rage au Laboratoire central d'élevage « $G$. Curasson » à Dakar, nous avons recherché, parmi les diverses méthodes possibles, celles qui étaient susceptibles de nous donner des indications précoces. Selon VILLEMOT et PROVOST (1-2), la précipitation en milieu gélifié est fidc̀le, sensible et permet d'effectuer un diagnostic dans les meilleurs délais.

Nous avons, pour notre part, appliqué cette technique, selon les indications de ces auteurs, sans obtenir de résultat satisfaisant sur plus de 30 animaux enragés appartenant à plus de dix espèces différentes. Devant cef échec, nous avons recherché les facteurs susceptibles d'influencer la réaction. L'exposé de leur étude va faire l'objet de la présente note. Nous comparerons ensuite la valeur de la réaction de précipitation d'Ouchterlony et Oudin à celle des autres méthodes. Rappelons toutefois que les expériences ont été conduites à Dakar sur des animaux de la région. Ce point, on le verra, est important à considérer.
\end{abstract}

\section{TECHNIQUE}

La réaction de précipitation en milieu gélifié, selon la modification de MANSI (3) à la technique d'Ouchterlony et Oudin décrite par VILLEMOT et PROVOST (1-2), consiste à mettre en présence dans un milieu gélifié un antigène et un antisérum. Les lignes de précipitation apparaissent dans les 48 heures. Pour déterminer les condi-

Reçu pour publication : novembre 1960.

Rev. Elev. Méd. vét. Pays trop. 1960, 13, n³ 4. tions de milieu les plus favorables, il convient donc de mettre en présence dans un milieu donf la composition sera variable, d'une part un antigène ef l'antisérum réagissant favorablement, d'autre part les mêmes éléments réagissant mal ou pas du tout.

\section{Choix de l'antigène}

L'antigène, ainsi qu'il est bien connu, a d'autant plus de chances de donner des lignes de précipitation que la teneur en substance antigénique est suffisante. Des essais antérieurs nous ont appris que le cerveau de lapin infecté avec le virus rabique fixe donne deux arcs de précipitation bien visibles en regard du sérum antirabique purifié de l'Institut Pasteur de Paris, épuisé par une suspension de cerveau de lapin normal. Le cerveau de lapin normal donne à lui seul 4 à 6 lignes de précipitation dans les conditions les plus favorables. Le cerveau de quelques chiens morts de l'infection par le virus des rues donne une réaction lente et toujours faible. Pour nos essais, nous nous sommes donc servi de cerveau de lapin mort de rage à virus fixe et de cerveaux de deux chiens morts de rage à virus des rues, l'un donnant un arc de précipitation et l'autre pas.

\section{$2^{\circ}$ Choix du sérum antirabique}

Les lignes de précipitation les plus nettes étant obtenues avec le sérum antirabique purifié de l'Institut Pasteur de Paris, nous nous sommes servi de ce sérum conjointement avec un sérum antirabique que nous avons obtenu sur un chien ef à un sérum normal de lapin. 


\section{Etude du milieu gélifié}

Il est habituellement recommandé d'employer pour les réactions de précipitation une gélose très pure. N'ayant à notre disposition qu'un agar de bonne qualifé $\left({ }^{*}\right)$, nous l'avons purifié de la manière suivante : trois lavages à l'acétone, puis séchage de 24 heures à l'étuve à $37^{\circ} \mathrm{C}$. Dix lavages à l'eau distillée à la température du laboratoire (après agitation violente dans un ballon rempli aux Irois quarts d'eau distillée, la gélose est laissée pour sédimentation. Siphonage de l'eau que l'on remplace par de l'eau distillée. L'opération s'effectue en deux jours). L'eau est alors filtrée et l'agar est séché à l'étuve à $37^{\circ} \mathrm{C}$. On fait alors, au bain-marie à $100^{\circ} \mathrm{C}$, un gel à 3 p. 100 et un autre à 4 p. 100 dans lequel on dissout du merthiolate de sodium pour obtenir une concentration de 3 p. 10.000 .

Ces géloses sont conservées en frigidaire en attendant l'emploi. A partir d'elles, nous avons confectionné une série des milieux à 1,5 p. 100 et 2. p. 100 de gélose en mélangeant à parties égales, au bain-marie, avec :

1) Chlorure de sodium .......... $16 \mathrm{~g}$

Rouge Congo ..............

Eau distillée .............. $1.000 \mathrm{ml}$

ce qui constitue la gélose préconisée par MANSI, puis VILLEMOT ef PROVOST.

2) un tampon phosphate à $\mathrm{pH} 6$ de concentration double.

3) un tampon phosphatc à $\mathrm{pH} 7$ de concentration double.

4) un tampon véronal à $\mathrm{pH} 8$ de concentration double.

5) un tampon véronal à $\mathrm{pH} 8,6$ de concentration double.

Les milieux obtenus après filtration sont coulés dans des boîtes de Pétri et des petits puits distants de $7 \mathrm{~mm}$ sont creusés, selon la méthode de Villemot et Provost.

A la suite de cette première série d'observations faites à la température du laboratoire $\left(24^{\circ}-26^{\circ} \mathrm{C}\right)$, nous avons constaté, ainsi que I'on devait s'y attendre, que les arcs de précipitation appara issaient plus précocement en milieu légèrement alcalin. Le $\mathrm{pH} 6$ s'est montré net-

(*) Bacloagár Difco. tement défavorable, le $\mathrm{pH} 7$ acceptable, le $\mathrm{pH} 8$ a permis l'obtention d'un précipité précoce, le $\mathrm{pH} 8,6$ donnant des résultats peu différents de ceux que donne le $\mathrm{pH} 7$. Toutefois le milieu tamponné paraît préférable au milieu non tamponné. En outre, la concentration de gélose à 1,5 p. 100 permet une diffusion plus précoce el une lecture plus rapide que la gélose à 2 p. 100. Cependant cette gélose ne s'oppose pas à la réaction, ce qui est important lorsqu'on désire effectuer la réaction d'immuno-électrophorèse, comme nous le préciserons ci-dessous.

Afin de déterminer le $\mathrm{pH}$ optimum, nous avons fait une série de géloses dont le $\mathrm{pH}$ variait de 0,2 en 0,2 et qui s'étendait de $\mathrm{pH} 7$ à $\mathrm{pH} 8,6$. En même temps, pour déterminer l'influence de la nature du tampon, nous avons employé des tampons phosphate $\left(^{*}\right)$ (phosphate monosodique $\frac{M}{10}+$ phosphate disodique $\frac{M}{10}$ et des tampons véronal $\left(^{* *}\right)$ (Véronal sodique $\frac{M}{10}+\mathrm{HCl}$ ).

La précipitation a été la plus précoce et la plus nette au $\mathrm{pH} 8$ en tampon phosphate, légèrement retardée au $\mathrm{pH} 8$ en tampon véronal. Nous avons ainsi obtenu en moyenne 4 à 6 lignes de précipitation, tant avec le cerveau de lapin normal qu'avec le cerveau de lapin enragé vis-à-vis du sérum antirabique purifié de l'Institut Pasteur de Paris. Les premières lignes de précipitation apparaissent après 12 à 18 heures, ce qui constitue un gain de temps appréciable.

Ayant constaté, au cours de nos études antérieures, l'importance de l'épaisseur de la gélose pour qu'une bonne réaction se produise, nous avons fait ces premiers essais avec une épaisseur de gélose de $5 \mathrm{~mm}$. Contrôlant, dans ces conditions, l'épaisseur optimum pour obtenir des arcs de précipitation aussi bien précoces qu'intenses, une épaisseur de 5 à $6 \mathrm{~mm}$ donne de bons résultats, mais la lecture est plus commode lorsque l'épaisseur n'est que $5 \mathrm{~mm}$. En raison des variations de dimensions des boîtes de Pétri et de la forme du fond, il est préférable de mesurer pour chaque modèle de boîte la quantité de gélose que l'on doit couler.

La réaction n'esf pratiquement pas influencée

(*) Table de $S \varnothing$ RENSEN.

(**) Table de MICHAELIS. 
par la température lorsqu'on se trouve en milieu tropical. La température moyenne du laboratoire variant de 20 à $28^{\circ} \mathrm{C}$ ne modifie pas la précocité et l'intensité de la réaction. Une chaleur de $370^{\circ} \mathrm{C}$ produit, au contraire, une intense condensation gênante sur les parois de verre des boîtes de Pétri.

\section{$4^{0}$ Choix de la méthode}

Nous avons effectué nos premières réactions selon la technique de Villemot et Provost, c'està-dire dans des boîtes de Pétri, les cupules dans la gélose de $7 \mathrm{~mm}$ de diamètre étant séparées de $7 \mathrm{~mm}$. Par la suite, nous avons employé une microréaction donnant des indications plus précoces encore et ne nécessitant qu'une quantité infime tant de matériel virulent que de sérum.

Pour ce faire, on coule $2 \mathrm{ml}$ de gélose tamponnée sur une lame porte-objet normale $(26 \times 27)$. Après refroidissement, on creuse avec un tube à paroi mince et en aspirant de petites cupules de 2 et $3 \mathrm{~mm}$ de diamètre réparties de la façon suivante qui s'est révélée la meilleure: une cupule centrale de $2 \mathrm{~mm}$ destinée au sérum (anticorps) et 4 à 6 cupules de $3 \mathrm{~mm}$ dont le centre est situé à $5 \mathrm{~mm}$ du centre de la cupule du sérum. Les cavités périphériques recevront les cerveaux (antigènes). Le sérum est versé avec une pipette capillaire. II n'est pas besoin, en général, d'effectuer plusieurs remplissages. Le cerveau finement broyé avec de petits ciseaux courbes et pointus est déposé dans les cupules périphériques. Les lames sont conservées en atmosphère humide dans des bacs ou boîtes de Pétri renfermant un coton movillé.

La réaction s'effectue en 8 à 12 heures. Après 24 heures toutes les lignes de précipitation sont apparves.

Toutes les réactions ont été faites avec ces deux méthodes. La microméthode nous a paru la meilleure, car plus précoce et aussi lisible.

\section{RÉSULTATS}

\section{lo Spécificité de la réaction}

Connaissant les conditions qui permettent d'obtenir des arcs de précipitation précoces et intenses, il est indispensable d'en étudier la spécificité, d'autant plus qu'il est très difficile de diagnostiquer la rage chez le lapin par le seul examen des lignes de précipitation souvent en nombre semblable chez les témoins ef les animaux affectés.

Effectuant la réaction avec les sérums antirabiques, purifiés ou bruts, nous avons obtenu avec le cerveau de douze lapins normaux de 4 à 6 lignes de précipitation. Ces sérums épuisés. avec des broyats de cerveaux des lapins renfermant le plus d'antigènes, ne donnent plus de: lignes avec les cerveaux de lapins normaux. mais deux lignes de précipitation avec le cerveau de lapins enragés. Ils paraissent spécifiques en ce sens que l'on n'obtient la réaction qu'avec des. animaux enragés. Tandis que le cervequ d'un bouc mort de rage à virus fixe donnait quatre lignes de précipitation avec le sérum antirabique. brut, ces lignes tombaient à deux avec le sérum épuisé.

Nous avons cherché à effectuer la réaction: avec un sérum antirabique de chien ayant un pouvoir séro-neutralisant très élevé $(3,4)$. Nous. n'avons obtenu avec ce sérum aucune précipitation spécifique, mais seulement, ef ceci surtout avec le chat, des lignes très précoces et très. intenses dues à la présence dans l'encéphale de salmonelles ou de proteus. Nous avons fréquemment observé à Dakar la sortie, chez le chat. d'entérobactéries que l'on retrouve dans le système nerveux. Ce sont ces germes contre lesquels. le chien est, en général, naturellement immunisé, qui produisent de fausses réactions. Pour avoir toute garantie, le sérum servant à l'épreuve de. précipitation doił être éprouvé vis-à-vis des germes que le cerveau peut renfermer accidentellement.

On pourrait critiquer l'emploi du sérum antirabique hyperimmun purifié en raison des modifications qu'il a subies lors de la purification Cependant lorsque le sérum est épuisé avec le cerveau de lapin, la réaction est tout-à-fait satisfaisante. Bien plus, nous avons obtenu certaines réactions de précipitation avec le sérum purifié, alors que le sérum brut ne les donnait pas. L'extraction des globulines du sérum brut d̀. l'aide du sulfate d'ammonium suivi d'une dialyse, permet dans ces cas d'obtenir la réaction. II existe un véritable effet inhibiteur des albumines. Ce fait a été confirmé par l'immunoélectrophorèse dans laquelle le sérum brut séparé par électrophorèse a été mis au contact d'un broyat de cerveau. 


\section{$2^{\circ}$ Valeur de la réaction}

La réaction, si elle semble spécifique avec les précédentes réserves, est-elle constante? C'est ce qui conditionne son emploi dans la pratique courante du diagnostic de la rage.

La réaction a été faite avec du sérum hyperrimmun tant purifié que brut, vis-à-vis d'un grand nombre d'animaux enragés. Il s'agissait, chez le chien, de rage des rues naturelle et de rage expćrimentale à virus fixe. Pour les autres espèces animales, la rage était uniquement expérimentale et chaque espèce a été titrée avec le virus des rues et le virus fixe, squf les bovins et les équins pour lesquels le contrôle n'a été fait qu'avec le virus fixe.

La réaction effectuée à $\mathrm{pH} 7$ selon la méthode préconisée par Villemot et Provost, s'est montrée négative chez tous les animaux suivants: souris blanche, rat blanc, rat à capuchon, rat de Gambic (Cricetomys gambianus), rat palmiste (Euxerus erythropus), rat gris (Ratfus rattus), hérisson, chat, chacal, bouc à virus des rues, porc, veau, cheval. Autrement dit, nous n'avons obtenu de diagnostic positif qu'avec quelques très rares chiens, le bouc à virus fixe et le lapin, de très rares chats à virus fixe.

La même réaction à $\mathrm{pH} 8$ avec un tampon phosphate devient positive vis-à-vis de quelques autres chiens, et de quelques souris blanches.

La conservation du cerveau de lapin à une température de $-5^{\circ} \mathrm{C}$ à $-10^{\circ} \mathrm{C}$ modifie les antigènes en ce sens que les lignes de précipitation tendent à se rapprocher et certaines à disparaître après 2 à 5 mois selon les cerveaux. L'a conservation en glycérine du cerveau de lapin tend au contraire à entraîner un écartement des lignes de précipitation.

Ainsi la réaction de précipitation en milieu gélifié, bien que dovée d'une spécificité suffisante, ne peut servir au diagnostic de la rage dans l'ovest africain. Un certain nombre de conditions doivent être réalisées pour que la réaction soit positive. Nous allons passer en revue celles que nous avons pu mettre en évidence, car elles permettent dc concevoir sous un jour nouveau les antigènes rabiques.

\section{$3^{\circ}$ Qualifés que l'antigène doit posséder pour la précipitation}

En dehors des conditions de milieu sur lesquelles nous nous sommes déjà étendu, l'anti- gène peut dans certains cas donner la précipitation spécifique. Celle-ci apparaît, en effet, chez la souris pendant les mois de mai et juin principalement et jamais pendant les mois de septembre et octobre. L'étude des variations tissulaires selon la saison, que nous avons faite antérieurement (5), montre que la réaction se produit lorsque l'organisme possède au maximum ses qualités fonctionnelles. Ainsi le virus peut entrâner la mise en évidence chez un animal de l'antigène virulent, mais pas celle de l'antigène soluble précipitable. II y a dissociation entre ces deux qualités de l'antigène rabique.

Cette condition n'est pas à elle seule suffisante car, pendant cette pćriode, tous les animaux ne font pas d'antigène soluble précipitable. On constate qu'il faut également que le titre du virus soit suffisamment élevé. Si l'on compare la DL 50 sur souris des cerveaux de chien qui donnent une réaction dc prćcipitation à celle des cerveaux de chien qui n'en donnent pas, on constate que si la DL 50 est supérieure à $10^{-\mathbf{4}}$, ¡l peut y avoir précipitation alors qu'elle n'apparaît pas si le titre est inférieur à 10-4. C'est ce qui explique aisément l'irrégularité de la réaction et sa constance chez le lapin infecté avec le virus fixe. Ceci est très net chez la souris infectée par passage en série avec le virus fixe passé sur lapins. La réaction apparaît chez un certain nombre dc sujets à partir du deuxième ef toujours à partir du troisième passage. Mais cette réaction devient rarement positive en dehors de la période favorable.

A la fin de la période des pluies, le titre du virus dans l'ovest africain est de l'ordre de DL $50=10^{-3}$ à $10^{-3}, 5$, ce qui, en outre, est insuffisant pour donner une bonne précipitation.

\section{$4^{\circ}$ Comparaison de la réaction de précipitation aux autres méthodes de diagnostic}

Pour CHABAUD, SÉRIÉ ef ANDRAL (6), l'électrophorèse du sérum est une méthode d'appoint intéressante pour le diagnostic de la rage. Dans l'ouest africain, elle est très inconstante. L'augmentation de la proportion des $\alpha_{2}$-globulines ne s'observe dans la rage que pendant la même période de mai-juin. Nous avons effectué l'électrophorèse du sérum de tous les animaux qui ont servi à l'étude de la précipitation en milieu gélifié, sans abtenir de résultat satisfaisant. Par comparaison avec la maladie de Carré du chien, 
on trouve dans cette maladie une variation également très faible des $x_{2}$-globulines, mais un fait intéressant est apparu. Seuls les chiens dont le sérum sanguin est très pauvre ou dépourvu de $\gamma$-globulines extériorisent les formes nerveuses. Il y a là une indication thérapeutique intéressante, soif pour les prévenir, soit pour abandonner un traitement qui se révélera par la suite voué à l'échec.

Il semble donc que pour l'électrophorèse. comme pour la précipitation en milieu gélifié. la méthode, quoique valable, ne puisse être appliquée partout.

Nous n'avons pas effectué la déviation du complément en raison des résultats défavorables obtenus par DEPOUX et MERVEILLE (7-8) dans la région de Brazzaville. II est vraisemblable que leurs échecs sont dus à des conditions particulières aux animaux étudiés, puisque cette même réaction donne des indications excellentes en d'autres points du globe.

Nous avons cherché par l'immunoélectrophorèse à améliorer la réaction de précipitation en milieu gélifié. Nous avons utilisé une gélose à 2 p. 100 de $\mathrm{pH} 8$ avec une rainure centrale de $1 \mathrm{~mm}$ de largeur destinée au sérum antirabique. séparée de $5 \mathrm{~mm}$ des cupules où l'on place l'antigène. On peut ainsi très facilement identifier 4 arcs de précipitation dans le cerveau de lapin normal et 5 dans le sérum de ce même animal. Le sérum antirabique purifié renferme donc un nombre notable d'anticorps antilapin d'une part, anticerveau de lapin d'autre part. Avec le cerveau de lapin affecté avec le virus fixe, il n'apparaît pas d'arc de précipitation supplémentaire. Cette méthode ne paraît pas valable pour l'ovest africain, ce qui ne veut pas dire qu'elle ne peut rendre des services ailleurs. Elle nous a en effet montré le pouvoir inhibiteur dans certains cas des albumines sériques lorsque l'on faisait l'électrophorèse du sérum brut non purifié et que l'on mettait en diffusion du cerveau de lapin infecté.

L'histopathologie de la rage conserve donc toute sa valeur en ouest africain dans le diagnostic chez le chien, puisque même en l'absence de corps de Négri, on trouve toujours chez cet animal une forte infiltration leucocytaire avec présence de nodules de Van Gehuchten et Nelis dans les ganglions de Gasser. Les autres affections ne donnent au maximum qu'une très dis- crète infiltration cellulaire et de très rares images de neuronophagie. En l'absence de toute lésion de ces ganglions, on peut conclure à l'absence de rage.

L'inoculation oux animaux d'expérience est, d̀ condition d'employer un nombre suffisant d'animaux très jeunes, la méthode de contrôle indispensable en tenant compte du fait que parfois le virus peut sortir après un délai très long, jusqu'à trois mois chez la souris, d'où la nécessité de l'inoculation d'un grand nombre d'animaux. On peut, toutefois, faire sortir cette rage latente par l'inoculation sous-cutanée d'un virus fixe inactivé.

\section{DISCUSSION}

Les résultats de la précipitation en milieu gélifié pour le diagnostic de la rage obtenus en ovest africain, s'ils sont décevants quant à l'utilisation d'une nouvelle méthode de diagnostic, sont intéressants parce qu'ils permettent de distinguer l'antigène infectant de l'antigène soluble précipitable. Ce dernier n'est pas indispensable pour l'infection. Joue-t-il un rôle dans la forme clinique de la rage? Est-il en rapport avec le fait que la rage en ovest africain est presque toujours de type paralytique? II est, pour l'instant, impossible de répondre à ces questions.

L'absence d'antigène précipitant va retentir sur la qualité du sérum hyperimmun que l'on peut obtenir dans cette région. En effet, en injectant régulièrement à un chien une suspension de cerveau de chien renfermant soit du virus rabique fixe, soit du virus rabique des rues, on obtient un sérum hyperimmun d'un titre neutralisant normal, mais dépourvu de pouvoir de précipitation pour des cerveaux précipités par le sérum antirabique purifié. II en est de même chez le cobaye. Ceci montre bien l'absence de cette fraction ontigénique, alors que l'animal est capable d'élaborer des anticorps neutralisants.

Cette constatation est en parfait accord avec les conceptions de KIPPS et Coll. (4), puisque la séparation mécanique qu'ils obtiennent des particules virales ef de l'antigène soluble est ici réalisée par l'organisme qui n'assure la synthèse que d'une seule fraction. II est toutefois curieux de noter que l'anticorps précipitant qui paraît le plus résistant aux agents physiques ou chimiques, soit celui que l'organisme n'élabore pas, 
comme si la résistance correspondait à une complexité plus grande ou à un besoin énergétique supérieur pour sa synthèse.

Le fait que l'on puisse obtenir un virus sans antigène soluble chez de nombreuses espèces animales, aussi bien chez les jeunes que chez les adultes, plaide contre l'hypothèse du précurseur antigénique du virus.

Nous ne pouvons, pour l'instant, interpréter d'une manière valable l'obligation d'un titre de virulence élevé pour qu'apparaisse l'antigène soluble.

\section{CONCLUSION}

La méthode de précipitation en milieu gélifié ne peut servir au diagnostic de la rage dans l'ovest africain en raison de son inconstance. Cependant, l'apparition, à certaines périodes de l'année, d'un antigène soluble précipitable montre bien la dissociation des particules virales et de l'antigène soluble. De même, on peut séparer l'anticorps neutralisant de l'anticorps précipitant.

Cetfe constatation va de pair avec l'irrégularité de l'augmentation des $\alpha_{2}$-globulines du sérum.

Ceci doit inciter à une grande prudence lorsque l'on obtient des résultats différents de ceux qu'ont publiés les promoteurs d'une méthode. II ne s'agit pas toujours d'une question de technique, mais de réactifs particuliers.

Travail du Laboratoire Central de l'Elevage «G. CURASSON » Directeur P. MORNET.

\section{BIBLIOGRAPHIE}

1. VILLEMOT (J. M.) et PROVOST (A.). - Précipitation spécifique du virus rabique en milieu gélifié selon la méthode d'OudinOuchterlony (Technique de Mansy). 'R. Acad. Sci., 1958, 246 : 2694-5.

2. VILLEMOT (J. M.) et PROVOST (A.). - Précipitation en milieu gélifié du virus rabique par le sérum hyperimmun. Revi Elev. Méd. vét. Pays trop., 1958, II : 387-97.

3. MANSI (W.). - The Study of some virus by the slide gel diffusion precipiting test. J. comp. Path., 1957, $67:$ 297-303.

4. KIPPS (A.), DU T. NAUDE (W.), POLSON (A.), SELZER (G.) et VAN DEN ENDE (M.). - The size distribution of specific antigens in virus infected tissues and their significance. Ciba Foundation Symposium on the nature of viruses, 1957.

5. THIERY (G.). - Etude des variations tissulaires saisonnières de quelques espèces animales vivant dans la région de Dakar. Rev. Elev. Méd. vét. Pays trop., 1959, 12 : 273-92.

6. CHABAUD (M. A.), SERIE (Ch.) et ANDRAL (L.). - Electrophorèse ef diagnostic de la rage. Ann. Inst. Past., 1955, $88: 420-34$.

7. DEPOUX (R.) et MERVEILLE (P.). - Sur la valeur de la réaction de déviation du complément dáns le diagnostic de la rage. Ann. Inst. Past., 1956, $90: 182-6$.

8. DEPOUX (R.) et ORIO (J.). - Deuxième note sur la réaction de fixation du complément dans le diagnostic de la rage. Bull Soc. Path. Exo., 1958, 51: 157-9.

\section{SUMMARY}

Variability of the results of the gel-diffusion method for the diagnostic of rabies in the region of Dakar.

Having tried out the gel-precipitation test on 30 positive cases of rabies at Dakar with inconsistent results, the author is of the opinion that the test is not suitable for West Africa. A combination of several factors is necessary in order to reach a positive result, these include (the environmental conditions?) the titre of the virus and the quality of the antigen. Only at certain seasons of the year at Dakar is a specific precipitation attainable. 


\section{RESUMEN}

Qué se puede esperar del método de precipitación en medio a base de ágar en el diagnóstico de la rabia en la región de Dakar.

Habiendo aplicado el método de precipitación sobre medio a base de ágar para el diagnóstico de la rabia en Dakar sobre más de 30 animales rabiosos, el autor, ante la inconstancia de los resultados obtenidos concluye que dicho método no es util en el œste africano. Numerosos factores deben agruparse para que esta reacción sea positiva. Los hay que dependen de las condiciones del medio, del tífulo del virus, de la calidad del antigeno. Se ha observado, que la precipitación especifica sobre algunas especies animales de la región, solamente se produce en ciertos periodos del año, debido a la aparición de un antígeno soluble precipitable. 